\title{
Aplicação de Técnicas de Mineração de Dados Baseada na Taxonomia de Bloom para o Mapeamento do Conhecimento na Aprendizagem de Programação
}

\author{
Rozelma Soares de França, Haroldo José Costa do Amaral ${ }^{1}$ \\ Universidade de Pernambuco, Campus Garanhuns (UPE) \\ Garanhuns, PE - Brasil \\ \{rozelma.soares, haroldo.amaral\}@gmail.com
}

\begin{abstract}
This article presents a model for application of mining techniques on data collected from assessments of learning. From the proposal, it becomes possible to identify which content and cognitive levels at which they were assimilated by the learners, thus allowing pedagogical strategies that are appropriately designed for groups of students who have difficulties identified. To validate the study, the results of an experiment conducted on a Programming discipline in Computer Science from the University of Pernambuco are also presented.
\end{abstract}

Resumo. Este artigo apresenta um modelo para aplicação de técnicas de mineração em dados coletados a partir de avaliações da aprendizagem. A partir da proposta, torna-se possível identificar quais conteúdos e em que níveis cognitivos eles foram assimilados pelos aprendizes, permitindo, assim, que estratégicas pedagógicas sejam adequadamente planejadas para grupos de estudantes que apresentem as dificuldades identificadas. Para validar o estudo, são apresentados também os resultados do experimento realizado numa disciplina de Programação do curso de Licenciatura em Computação da Universidade de Pernambuco.

\section{Introdução}

A avaliação é essencial à educação e indissociável enquanto concebida como problematização, questionamento e reflexão sobre a ação (HOFFMANN, 2010). Nessa perspectiva, ela desempenha um papel fundamental em promover a aprendizagem, produzindo informações que poderão ajudar estudantes e professores. Assim, a avaliação deixa de ser apenas um instrumento de verificação da aprendizagem e passa a atuar diretamente no processo de ensino e aprendizagem, permeando-o e auxiliando-o não mais como uma atividade em momentos estanques e pontuais (CERNY, 2001).

Há uma busca por uma avaliação formativa que pode ser entendida como uma prática de avaliação contínua, cujo objetivo é melhorar a aprendizagem em curso, contribuindo para o acompanhamento e orientação dos estudantes durante todo seu processo de formação (PERRENOUD, 1999). O processo de avaliação contínua pode produzir uma grande massa de dados, requerendo procedimentos automáticos ou semiautomáticos para o tratamento e análise dos dados. Nesse contexto, pesquisas vêm

\footnotetext{
${ }^{1}$ Orientador do Trabalho de Conclusão de Curso.
} 
sendo realizadas, investigando possibilidades de exploração dessas informações, através da utilização de técnicas de mineração de dados (ROMERO \& VENTURA, 2007).

Pimentel et al. (2006) e Santos et al. (2012) relatam a aplicação de técnicas de mineração em dados obtidos a partir de avaliações da aprendizagem. Porém, em seus estudos, não se observa, durante a seleção dos dados, a inclusão de atributos que façam referência aos objetivos pedagógicos planejados a serem alcançados pelos aprendizes. Assim, este artigo, fruto de um Trabalho de Conclusão do Curso de Licenciatura em Computação da Universidade de Pernambuco, tem como objetivo (re)apresentar um conjunto de atributos, elaborado a partir de uma taxonomia com fins educacionais, a Taxonomia de Bloom (BLOOM et al., 1956; ANDERSON \& KRATHWOHL, 2001), e a aplicação de técnicas de mineração de dados em avaliações contínuas da aprendizagem, que viabilize realizar o mapeamento do conhecimento de estudantes.

A partir da proposta, torna-se possível identificar quais conteúdos e em que níveis cognitivos eles foram assimilados pelos aprendizes, permitindo, assim, que estratégicas pedagógicas sejam adequadamente planejadas a grupos de estudantes que apresentem as dificuldades identificadas. Para validar o estudo, são apresentados os resultados da aplicação de três técnicas de mineração sobre dados coletados a partir de avaliações de aprendizagem provenientes de uma disciplina de Programação.

O restante do artigo está organizado como segue: a Seção 2 apresenta conceitos de mineração de dados e trabalhos relacionados no âmbito da educação. A Seção 3 apresenta um modelo para aplicação de técnicas de mineração em dados coletados a partir de avaliações da aprendizagem. A seção 4 descreve um experimento realizado e seus resultados. A Seção 5 apresenta a análise dos padrões encontrados. Por fim, na Seção 6, são feitas algumas considerações acerca deste trabalho, incluindo as contribuições da pesquisa realizada e direcionamentos para trabalhos futuros.

\section{Mineração de Dados Educacionais}

Com o avanço das tecnologias computacionais, que permitem o armazenamento e o processamento de um grande volume de dados, novas tecnologias têm sido desenvolvidas para auxiliar na extração de informações dessas bases de dados, através de técnicas, como a Descoberta de Conhecimento em Bases de Dados (Knowledge Discovery in Database KDD) e a Mineração de Dados (Data Mining - DM). Segundo Fayyad (1996), KDD consiste num processo de várias etapas, não trivial, interativo e iterativo, para identificação de padrões compreensíveis, válidos, novos e potencialmente úteis. Por outro lado, o processo de DM restringe-se à aplicação de algoritmos para extração de padrões (FAYYAD et al., 2002).

Técnicas de mineração de dados podem ser aplicadas a uma variedade de contextos de tomada de decisão, podendo, também, minerar dados de alunos para verificar a relação entre uma abordagem pedagógica e o seu aprendizado e, através dessa informação, o professor poder compreender se sua abordagem está realmente ajudando o estudante e desenvolver métodos de ensino mais eficazes (BAKER et al., 2011). Em sistemas educacionais, o conhecimento descoberto pode ser útil tanto para educadores e profissionais responsáveis pela concepção, planejamento, construção e manutenção dos sistemas, quanto para os próprios estudantes que interagem com a aplicação. Romero \& Ventura (2007) afirmam que a descoberta do conhecimento pode ser orientada a diferentes atores de acordo com os interesses particulares de cada um: 
- Orientada para estudantes: técnicas de mineração de dados são utilizadas para recomendar atividades, recursos e tarefas que favoreçam e melhorem a aprendizagem do aluno. Isso pode ser feito por meio de sugestões de links, baseadas nas experiências de aprendizagem de outros estudantes, por exemplo;

- Orientada para educadores: objetiva fornecer feedback aos instrutores, avaliar a estrutura do conteúdo do curso e a sua eficácia no processo de aprendizagem. Também é foco de interesse classificar grupos de estudantes baseado em suas necessidades, descobrir padrões de aprendizagem regulares e irregulares, descobrir os erros frequentes, descobrir atividades que são mais eficazes, organizar o conteúdo de forma mais eficiente para o progresso do aluno;

- Orientada para gestores educacionais: objetiva ter parâmetros para melhorar a eficiência do sistema e adaptá-lo ao comportamento de seus usuários, ter medidas sobre como melhor organizar os recursos institucionais (humanos e materiais), melhorar a oferta de programas educacionais, entre outros.

Há na literatura diversos trabalhos sobre mineração de dados educacionais. Como eixos de pesquisa podem-se destacar: i) criação de alertas sobre perfis de alunos com risco de evasão ou reprovação (KAMPFF et al., 2008); ii) relação entre colaboração e a aprendizagem de estudantes em ambientes de educação a distância (PRATA et al., 2009); iii) agrupamento de alunos pelos problemas de aprendizagem (CORREIA et al., 2011); iv) ferramentas de mineração de dados de fácil uso destinadas a educadores e não especialistas na área (PEDRAZA-PEREZ et al.,2011; MERCERON et al., 2005); v) modelo automatizado para identificação de comportamentos inadequados em sistemas educacionais (BAKER et al., 2006).

\section{Uma Abordagem Inovadora para Mineração de Dados Educacionais}

Para a definição e estruturação do modelo que viabilize realizar o mapeamento do conhecimento de estudantes, um conjunto de atributos possíveis de serem extraídos de disciplinas em curso é proposto neste trabalho. O Quadro 1 apresenta tais atributos, junto com as suas descrições, tipos e domínio de valores que eles podem assumir.

Considerando que a clara e estruturada definição de objetivos educacionais pode direcionar o processo de ensino para a escolha adequada de estratégias, métodos, delimitação do conteúdo específico, instrumentos de avaliação e, consequentemente, para uma aprendizagem efetiva e duradoura (FERRAZ et al., 2010), a abordagem proposta foi elaborada a partir de uma taxonomia de objetivos educacionais, a Taxonomia de Bloom (BLOOM et al., 1956; ANDERSON \& KRATHWOHL, 2001).

A Taxonomia de Bloom é composta por três domínios: Cognitivo, Afetivo, Psicomotor; entretanto, o domínio cognitivo é o mais conhecido e utilizado. No domínio cognitivo, os objetivos foram agrupados em seis diferentes categorias e apresentados numa hierarquia de complexidade. $\mathrm{Na}$ proposta original, as categorias desse domínio são: Conhecimento, Compreensão, Aplicação, Análise, Síntese e Avaliação. Porém, com a incorporação de novos conceitos, recursos e teorias ao campo educacional, bem como os avanços psico-pedagógicos e tecnológicos, e a publicação de diversas experiências de sucesso, no uso efetivo da taxonomia, os pressupostos teóricos que sustentam a pesquisa original apresentada em 1956 foram reavaliados e, assim, a Taxonomia de Bloom foi revisada e atualizada (ANDERSON \& KRATHWOHL, 2001). 
$\mathrm{Na}$ taxonomia revisada, a base original das categorias foi mantida; todavia, ao separar, conceitualmente, o conhecimento do processo cognitivo, ocorreram as seguintes mudanças (KRATHWOHL, 2002): i) os aspectos verbais utilizados na categoria Conhecimento foram mantidos, mas essa foi renomeada para Lembrar; ii) Compreensão foi renomeada para Entender; iii) Aplicação, Análise e Avaliação, foram alteradas para a forma verbal Aplicar, Analisar e Avaliar, respectivamente, por expressarem melhor a ação pretendida e serem condizentes com o que se espera de resultado a determinado estímulo de instrução; iv) Síntese trocou de lugar com Avaliação (Avaliar) e foi rebatizada para a forma verbal Criar; v) os nomes das subcategorias existentes foram alterados para verbos no gerúndio.

Para identificar o nível cognitivo em que os conteúdos de uma disciplina específica foram assimilados pelos estudantes, as categorias da Taxonomia de Bloom revisada foram incorporadas ao modelo aqui proposto.

Quadro 1. Atributos propostos para o mapeamento do conhecimento do estudante

\begin{tabular}{|c|c|c|c|}
\hline Atributo & Descrição & Tipo de Dado & Domínio \\
\hline IdAluno & $\begin{array}{l}\text { Código que identifica o aluno participante da sessão } \\
\text { de avaliação }\end{array}$ & Numeric & {$[1, \mathrm{n}]$} \\
\hline IdSessao & $\begin{array}{l}\text { Código que identifica o número da sessão de } \\
\text { avaliação e a sequência temporal }\end{array}$ & Numeric & {$[1, n]$} \\
\hline Idltem & $\begin{array}{l}\text { Código que identifica uma questão numa avaliação } \\
\text { formativa }\end{array}$ & Nominal & {$\left[Q_{01}, Q_{n}\right]$} \\
\hline Conteudo & Conteúdo tratado no item avaliado & Nominal & {$\left[\mathrm{C}_{01}, \mathrm{C}_{\mathrm{n}}\right]$} \\
\hline NivelCognitivo & $\begin{array}{l}\text { Nível cognitivo do item avaliado de acordo com a } \\
\text { Taxonomia de Bloom }\end{array}$ & Nominal & $\begin{array}{l}\text { LEM - Lembrar } \\
\text { ENT - Entender } \\
\text { APL - Aplicar } \\
\text { ANA - Analisar } \\
\text { AVA - Avaliar } \\
\text { CRI - Criar }\end{array}$ \\
\hline Conceitoltem & Grau de acerto do aprendiz no item avaliado & Nominal & $\begin{array}{l}\text { A }-8 \geq \text { nota } \leq 10 \\
B-6 \geq \text { nota }<8 \\
C-4 \geq \text { nota }<6 \\
D-2 \geq \text { nota }<4 \\
\text { E }-0 \geq \text { nota }<2 \\
?-\text { Ausência de informação }\end{array}$ \\
\hline ConceitoAvaliacao & $\begin{array}{l}\text { Nível de desempenho do aprendiz numa } \\
\text { determinada avaliação formativa }\end{array}$ & Nominal & $\begin{array}{l}\text { A }-8 \geq \text { nota } \leq 10 \\
B-6 \geq \text { nota }<8 \\
C-4 \geq \text { nota }<6 \\
D-2 \geq \text { nota }<4 \\
\text { E }-0 \geq \text { nota }<2 \\
?-\text { Ausência de informação }\end{array}$ \\
\hline RotuloSomativa & Rótulo da avaliação somativa & Nominal & $\begin{array}{l}\text { APR - Aprovado } \\
\text { REP - Reprovado }\end{array}$ \\
\hline
\end{tabular}

A partir do conjunto de atributos do Quadro 1, algumas questões podem ser formuladas na tentativa de descobrir conhecimento através dos algoritmos de reconhecimento de padrões. Eis alguns exemplos:

- Existe relação entre os níveis cognitivos da Taxonomia de Bloom e o desempenho do aprendiz nas avaliações?

- Existe relação entre o desempenho do aprendiz e a sequência cognitiva com que um conteúdo é trabalhado: Os estudantes apresentam melhor desempenho, em determinado conteúdo, quando o mesmo é trabalhado primeiramente no nível Aplicar e posteriormente no nível Entender?

- Existe relação entre o desempenho do aprendiz nas avaliações formativas e o rendimento na avaliação somativa?

- Que agrupamentos homogêneos de estudantes podem ser identificados a partir de seus desempenhos numa disciplina específica? 


\section{Experimentos e Resultados}

Com o objetivo de verificar a adequação do conjunto de atributos proposto, realizaram-se experimentos, na disciplina Programação Orientada a Objetos, do curso de Licenciatura em Computação da Universidade de Pernambuco, ministrada no primeiro semestre de 2010, onde havia 33 estudantes matriculados. A análise das médias das avaliações somativas revelou uma taxa de sucesso de $24,24 \%$, demosntrando a dificuldade encontrada por esses alunos. A partir dessa realidade, passou-se a tentar identificar se, dentre os dados disponíveis com o histórico das avaliações formativas, haveria evidências que pudessem destacar os fatores que dificultaram a aprendizagem.

A abordagem adotada na disciplina foi além da avaliação convencional, baseada no rendimento alcançado pelo aluno em poucas provas, adotando-se avaliações formativas, ao longo do processo de aprendizagem. Dentro desse escopo, foram considerados os dados obtidos a partir de 11 sessões de avaliação: 5 listas de exercícios, 4 miniprovas e 2 projetos. Cada sessão era composta por " $n$ " enunciados de problemas, totalizando 890 exercícios a serem avaliados pelo professor. Todas essas atividades antecederam às duas avaliações somativas, ocorridas ao fim de cada bimestre, e a análise e interpretação desses dados podem ser exploradas a fim de fornecer um melhor discernimento sobre o conhecimento e dificuldades dos estudantes na aprendizagem.

As avaliações foram formuladas de modo a obter uma medida cognitiva, que deveria retratar o real desempenho do estudante na resolução de cada problema e seria resultado do processo de correção do professor. Após a análise dos enunciados dos problemas, foi possível classificá-los de acordo com os níveis da Taxonomia de Bloom. Pelo fato de as questões não terem sido formuladas, tendo como referência uma taxonomia de objetivos educacionais, uma limitação deste trabalho está na classificação, já que o professor-autor poderia ter um objetivo diferente do apresentado nos enunciados e ter equivocadamente utilizado um verbete de um nível cognitivo que não desejava avaliar naquele momento nos estudantes, levando, assim, à classificação de uma questão em um nível superior ou inferior ao desejado pelo docente.

Para a realização dos experimentos, a ferramenta WEKA, desenvolvida pela Universidade de Waikato da Nova Zelândia (WITTEN \& FRANK, 2005), foi utilizada. Além disso, tendo em vista os objetivos almejados, as tarefas de Classificação ${ }^{2}$, Associação ${ }^{3}$ e Agrupamento ${ }^{4}$ foram escolhidas. Para este trabalho, considera-se que o uso dessas tarefas é complementar e não competitivo. A escolha dos algoritmos, que implementam as técnicas utilizadas nas tarefas mencionadas, baseia-se nos casos de sucesso apresentados por trabalhos correlatos, destacados nas Seções 1 e 2, que demonstraram que os algoritmos J4.8, Apriori e K-means obtiveram um bom resultado.

\footnotetext{
${ }^{2}$ A classificação de dados consiste no processo de predizer a classe de um objeto segundo certos atributos de predição (HAN \& KAMBER, 2001).

${ }^{3}$ Segundo Carvalho (2000), uma regra de associação é definida como: se $\mathrm{X}$ então $\mathrm{Y}$ ou $\mathrm{X} \rightarrow \mathrm{Y}$, onde $\mathrm{X}$ e $\mathrm{Y}$ são conjuntos de itens no formato atributo-valor e $\mathrm{X} \cap \mathrm{Y}=\phi$. Diz-se que $\mathrm{X}$ é o antecedente da regra e $\mathrm{Y}$ é o consequente da regra. A meta é descobrir se $\mathrm{X}$ implica $\mathrm{Y}$.

4 A tarefa de agrupamento visa agrupar um conjunto de dados por algum critério de similaridade e, diferentemente da tarefa de classificação, não devem ter sido previamente classificados (LOPES, 2003).
} 


\subsection{Tarefa de Classificação}

Para esta tarefa, foi necessário escolher um atributo alvo e os atributos de predição. Assim, escolheu-se como atributo alvo o conceito obtido pelo aprendiz na avaliação, ou seja, o nível de desempenho do estudante numa determinada avaliação formativa: A, B, C, D, E ou ?. A questão por trás desta escolha foi: O que determina o desempenho do aprendiz numa avaliação formativa?.

$\mathrm{O}$ algoritmo da árvore de decisão escolhido foi o J4.8. A Figura 1 ilustra parte da árvore gerada com base nos 890 registros transformados a partir dos dados originais.

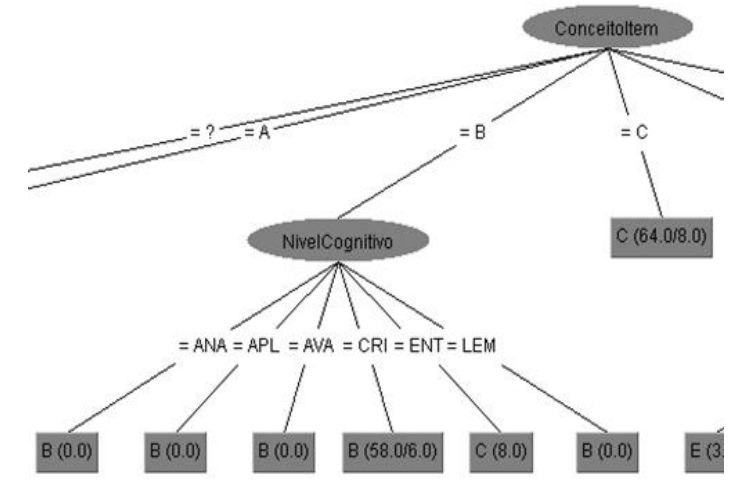

Figura 1. Parte da árvore de decisão gerada com o atributo alvo ConceitoAvaliacao

Analisando os padrões encontrados, podem-se ter as seguintes interpretações:

- Percebe-se que se o conceito obtido no item for igual a "B" (nota entre 6,0 e 8,0), então o conceito final na avaliação formativa dependerá do nível cognitivo em que o item foi classificado:

- Em 58 casos, exceto em 6, obtém-se conceito "B" na avaliação formativa se o item foi classificado no nível Criar;

- Em 8 casos, obtém-se conceito "C" na avaliação formativa se o item foi classificado no nível Entender.

- Em 64 ocorrências, exceto em 8, quando o estudante obtém o conceito "C" (nota entre 4,0 e 6,0) no item, ele tem desempenho final "C" na avaliação formativa.

Pode-se concluir que, pela árvore de decisão, o nível cognitivo em que o item é classificado interfere no desempenho final do aprendiz na avaliação formativa.

Após a descrição da árvore de decisão, outro atributo foi selecionado para análise. Dessa vez, escolheu-se como atributo alvo o rótulo na avaliação somativa (APR Aprovado ou REP - Reprovado) e procurou-se responder a seguinte questão: Existe relação entre o desempenho do aprendiz nas avaliações formativas e o rendimento na avaliação somativa?. A Figura 2 apresenta a nova árvore de decisão gerada.

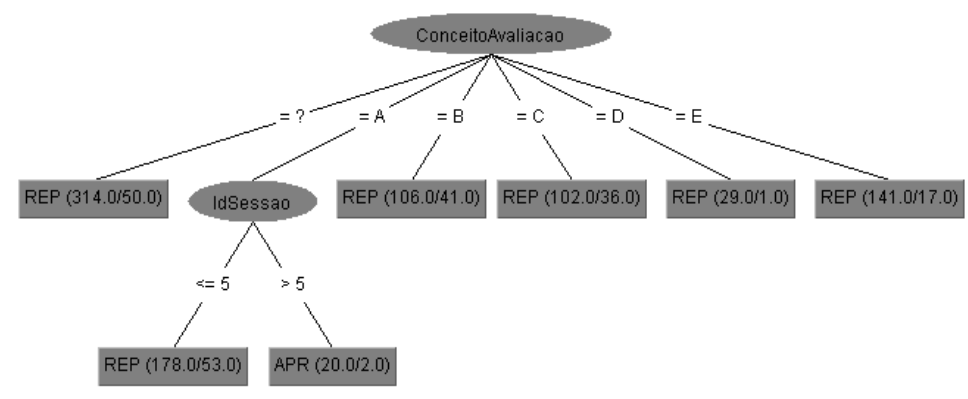

Figura 2. Árvore de decisão gerada com o atributo alvo RotuloSomativa 
Analisando-se os padrões encontrados pelo algoritmo J4.8, representados na árvore de decisão da Figura 2, pode-se realizar as seguintes leituras:

- Percebe-se que, quando o estudante obtém o conceito " $A$ " na avaliação formativa, o rótulo da avaliação somativa depende do ID da avaliação formativa, sendo:

- Rótulo APR (aprovado) se o ID da sessão de avaliação for superior a 5;

- Rótulo REP (reprovado) se o ID da sessão de avaliação for igual ou inferior a 5.

- Nota-se também que, se o aprendiz obtém conceito "?", "B”, “C”, “D” ou "E”, na avaliação formativa, o rótulo que ele recebe na avaliação somativa é REP.

Pode-se concluir que, com base nesses resultados, o desempenho pleno nas avaliações formativas $6,7,8,9,10$ e 11 é determinante para a aprovação do estudante. Outros atributos alvos podem ser selecionados para fins de análises comparativas.

\subsection{Tarefa de Associação}

A Figura 3 apresenta as 9 regras geradas pelo algoritmo de regras de associação Apriori. Analisando-se os padrões, as seguintes interpretações, dentre outras, podem ser feitas:

- Na regra 3, com confiança de 100\%, tem-se 132 casos que, quando o conteúdo é Estruturas de Repetição, o nível cognitivo explorado é o Criar;

- Na regra 4, com $100 \%$ de confiança, percebe-se que, quando o conteúdo é Estruturas de Repetição e o aprendiz obtém rótulo Reprovado na avaliação somativa, a abordagem de ensino fez uso do nível Criar;

- Na regra 8, com confiança de 93\%, tem-se que, quando o conteúdo é Arrays e não houve fornecimento de informação, pelo aprendiz, na resolução de um item, o nível cognitivo empregado no questionamento foi o Criar.

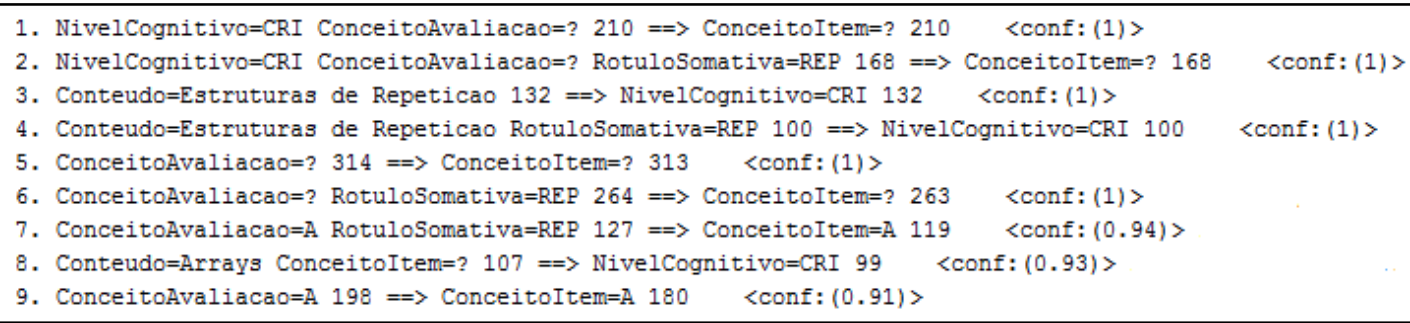

Figura 3. Regras de associação geradas pelo algoritmo Apriori

\subsection{Tarefa de Agrupamento}

Durante o treinamento foram feitos agrupamentos de 2 até 10 clusters (grupos). Identificouse que o agrupamento com 6 clusters resultou em centróides mais determinantes para formação de grupos homogêneos. A Figura 4 apresenta uma visão dos 6 grupos obtidos com o algoritmo de agrupamento (clusterização) K-means na ferramenta WEKA, gerados com base nos 890 registros transformados a partir dos dados originais da disciplina. 


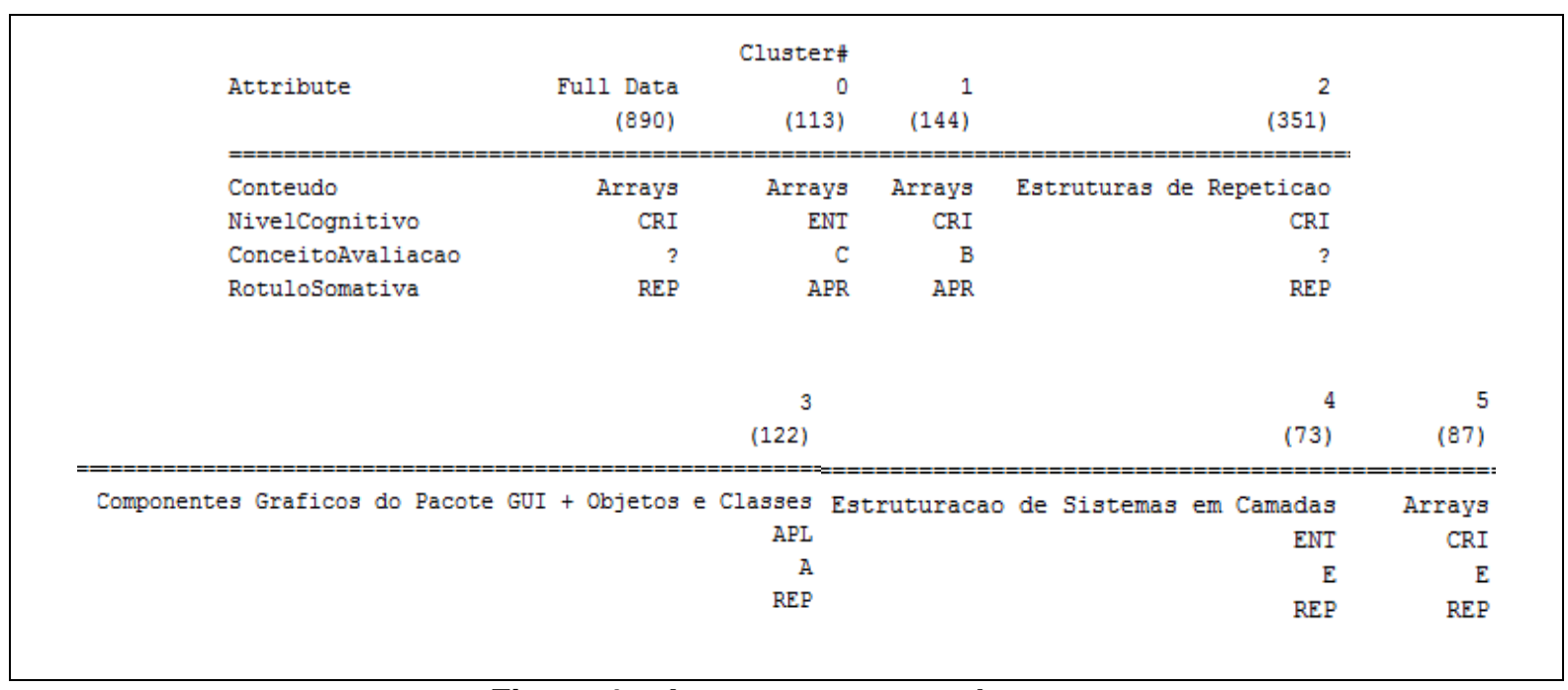

Figura 4 - Agrupamento em clusters

Analisando os clusters da Figura 4 é possível realizar algumas interpretações:

- Cluster 0: Neste cluster com 113 ocorrências de itens (13\%), foram agrupados os estudantes que alcançaram conceito "C" na avaliação formativa, composta por itens que tratavam sobre Arrays, abordados no nível Entender da Taxonomia de Bloom, e que obtiveram rótulo APR (aprovado) na disciplina;

- Cluster 1: Neste cluster com 144 ocorrências de itens (16\%), foram agrupados estudantes que alcançaram conceito "B" na avaliação formativa, composta por itens que abordavam o conteúdo Arrays, tratado no nível Criar da Taxonomia de Bloom, e que obtiveram rótulo APR (aprovado) na disciplina;

- Cluster 2: Este cluster agrupa estudantes reprovados, que não responderam às questões (351 itens, perfazendo 39\%) das avaliações formativas que tratavam sobre Estruturas de Repetição, as quais foram classificadas no nível Criar da Taxonomia de Bloom;

- Cluster 3: Este cluster agrupa estudantes que realizaram satisfatoriamente as questões sobre Componentes Gráficos do Pacote GUI e Objetos e Classes (14\% do total de itens), tratadas no nível Aplicar, mas que, todavia, foram reprovados;

- Cluster 4: Foram agrupados, neste cluster, estudantes que obtiveram baixo desempenho na resolução de itens ( $8 \%$ de 890$)$ que tratavam sobre Estruturação de Sistemas em Camadas que foram trabalhados no nível Entender. No final da disciplina, os alunos deste grupo foram rotulados como reprovados.

- Cluster 5: Representa estudantes com baixo desempenho na resolução de itens (10\% de 890$)$ de avaliações formativas que tratavam sobre Arrays, abordados no nível Criar. Os estudantes deste grupo foram rotulados como reprovados.

\section{Análise dos Padrões Encontrados}

Pela tarefa de Classificação, foi possível diagnosticar que existe relação entre os níveis cognitivos da Taxonomia de Bloom e o desempenho do aprendiz, bem como entre o desempenho do estudante nas avaliações formativas e o rendimento nas somativas.

Através da análise de padrões de regras de associação, constatou-se que um dos gargalos na aprendizagem da disciplina de Programação está no processo de criação (nível Criar da Taxonomia de Bloom), a exemplo de estruturas de repetição e arrays. Este fator 
atrelado a outros tem influenciado no aumento dos índices de reprovação da disciplina. $\mathrm{Na}$ abordagem associativa, o uso do algoritmo Apriori gerou regras que parecem "óbvias", sugerindo, assim, que outros algoritmos associativos sejam testados. No experimento descrito, o K-means foi utilizado. O uso desse algoritmo permitiu agrupar alunos pelas suas dificuldades de aprendizagem na disciplina. Foi possível, por exemplo, identificar um grupo de aprendizes com dificuldades em Entender Estruturação de Sistemas em Camadas e outro composto por estudantes com problemas em Criar Arrays. Ambos os grupos foram reprovados ao final do semestre.

Vale ressaltar que ainda não é possível concluir se os resultados obtidos com essas técnicas são significativos. Porém, as informações descobertas poderão ser utilizadas para apoiar as reuniões pedagógicas e a tomada de decisão que favoreçam a aprendizagem dos estudantes.

\section{Conclusões, Contribuições e Trabalhos Futuros}

Este trabalho apresentou uma abordagem inovadora para a identificação de problemas na aprendizagem pela aplicação de técnicas de mineração em dados provenientes de avaliações. Com os resultados, esta pesquisa demonstrou a viabilidade em se aliar técnicas de mineração de dados a objetivos educacionais, sendo importante para a elaboração de estratégias pedagógicas que favoreçam a aprendizagem de estudantes com tendência a não alcançar desempenho satisfatório. Assim, espera-se ter contribuído com a comunidade de Informática na Educação pela construção de um modelo conceitual que possibilita identificar o nível cognitivo em que determinado conteúdo foi assimilado por aprendizes de determinada disciplina; por estruturar a proposta de maneira a permitir a sua adequação nas diversas áreas do conhecimento e por viabilizar a aplicabilidade do modelo nas modalidades de ensino presencial e à distância.

Foram alcançados outros indicadores como parte do processo de pesquisa associado à realização deste trabalho de conclusão de curso, como: i) participação em eventos que discutem Informática na Educação e ii) publicação em conferência e revista nacionais (FRANÇA \& AMARAL, 2013a; FRANÇA \& AMARAL, 2013b). Como trabalhos futuros, pretende-se aprofundar os testes e análise dos padrões encontrados no estudo de caso realizado, bem como replicar o método proposto em outras turmas e avaliar a intervenção dos resultados. Somado a isso, planeja-se incorporar ao conjunto de atributos apresentado outros dados que carreguem informações a despeito das estratégias utilizadas pelos estudantes na resolução das atividades propostas.

\section{Referências}

Anderson, L. W.; Krathwohl, D. R. (Ed.) (2001). "A taxonomy for learning, teaching, and assessing: A revision of Bloom's taxonomy of educational objectives". New York: Addison Wesley Longman.

Baker, R. S. J. D.; Corbett, A. T.; Koedinger, K. R.; Evenson, S. E.; Roll, I.; Wagner, A. Z.; Naim, M.; Raspat, J.; Baker, D. J.; Beck, J. (2006) "Adapting to When Students Game an Intelligent Tutoring System". In: Proceedings of the International Conference on Intelligent Tutoring Systems, p. 392401.

Baker, R.; Isotani, S.; Carvalho, A. (2011). "Mineração de Dados Educacionais: Oportunidades para o Brasil". Revista Brasileira de Informática na Educação, v. 19, n. 02, p. 3-13.

Bloom, B.S. (Ed.); Engelhart, M. D.; Furst, E. J.; Hill, W. H.; Krathwohl, D. R.. (1956) "Taxonomy of educational objectives: The classification of educational goals. Handbook 1: Cognitive domain". New York: David McKay. 
Carvalho, J. V. (2000). "Reconhecimento de Caracteres Manuscritos Utilizando Regras de Associação". Dissertação de Mestrado. UFCG/COPIN.

Cerny, R. Z. (2001). "Uma reflexão sobre a avaliação formativa na educação a distância". UFSC.

Correia, C. F; Pimentel, E. P. (2011) "Mineração de Dados na Formação de Turmas para a Recuperação Paralela na Educação Básica". In: XXII Simpósio Brasileiro de Informática na Educação, Aracaju. Anais do XXII SBIE - XVII WIE, p. 172-175.

Fayyad, U.; Grinstein, G. G.; Wierse, A. (2002). "Information Visualization”. In: Data Mining And Knowledge Discovery. Morgan Kaufmann Publishers Inc, San Francisco, CA, USA.

Fayyad, U.; Piatetsky-Shapiro, G.; Smyth, P. (1996). "From Data Mining to Knowlegde Discovery". American Association for Artificial Intelligence.

Ferraz, A. P. do C. M.; Belhot, R. V.. (2010). "Taxonomia de Bloom: revisão teórica e apresentação das adequações do instrumento para definição de objetivos instrucionais”. Gest. Prod., São Carlos, v. 17, n. 2, p. 421-431.

França, R. S. ; Amaral, H. J. C. (2013a) "Aplicação de Técnicas de Mineração de Dados para o Mapeamento do Conhecimento na Aprendizagem de Programação: Uma Estratégia Baseada na Taxonomia de Bloom”. In: XXI Workshop sobre Educação em Computação (WEI), Maceió. Anais do XXXIII Congresso da Sociedade Brasileira de Computação.

França, R. S. ; Amaral, H. J. C. (2013b) "Mineração de Dados na Identificação de Grupos de Estudantes com Dificuldades de Aprendizagem no Ensino de Programação". RENOTE. Revista Novas Tecnologias na Educação, v. 11, p. 1-10.

Han, J.; Kamber, M. (2001). "Data Mining: Concepts and Tecniques". San Francisco: Morgan Kaufmann Publishers.

Hoffmann, J. M. L. (2010). "Avaliação: mito e desafio - uma perspectiva construtivista". Porto Alegre: Mediação.

Kampff, A. J. C.; Reategui, E. B.; Lima, J. V. de. (2008) "Mineração de dados educacionais para a construção de alertas em ambientes virtuais de aprendizagem como apoio à prática docente". Novas Tecnologias na Educação. v. 6, No 2, Dezembro.

Krathwohl, D. R. (2002). “A revision of Bloom's taxonomy: an overview. Theory in Practice”, v. 41, n. 4, p. 212-218.

Lopes, C. C. (2003) "Um Sistema de Apoio à Tomada de Decisão no Acompanhamento do Aprendizado em Educação a Distância". Campina Grande: UFCG. Dissertação de Mestrado.

Merceron, A.; Yacef, K. (2005) "TADA-Ed for Educational Data Mining”. Interactive Multimedia Electronic Journal of Computer-Enhanced Learning. Volume 7, Number 1, May.

Pedraza-Perez, R.; Romero, C.; Ventura, S. (2011) “A Java desktop tool for mining Moodle data”. In: Proceedings of the 4th International Conference on Educational Data.

Perrenoud, P. (1999). "Avaliação: da excelência à regulação das aprendizagens entre duas lógicas". Porto Alegre: Artes Médicas.

Pimentel. E. P.; Omar, N. (2006). "Descobrindo Conhecimentos em Dados de Avaliação da Aprendizagem com Técnicas de Mineração de Dados”. In: XII Workshop de Informática na Escola, Campo Grande, MS. Anais do XXVI CSBC.

Prata, D. N.; Baker, R. S. J. D.; Costa, E., Rosé, C. P.; Cui, Y.; Carvalho, A. M. J. B de. (2009). "Detecting and Understanding the Impact of Cognitive and Interpersonal Conflict in Computer Supported Collaborative Learning Environments". In: Proceedings of the International Conference on Educational Data Mining, p. 131-140.

Romero, C; Ventura, S.. "Educational Data Mining: A Survey from 1995 to 2005" (2007). Expert Systems with Applications 33, p. 125-146.

Santos, H. L. dos; Camargo, F. N. P.; Camargo, S. da S. (2012). "Minerando Dados de Ambientes Virtuais de Aprendizagem para Predição de Desempenho de Estudantes". In: Seventh Latin American Conference on Learning Objects and Technologies, Guayaquil. Proceedings of the 7th LACLO.

Witten, I. H.; Frank, E. (2005). "Data Mining: Practical Machine Learning Tools and Techniques". 2nd edition, Morgan Kaufmann Publishers, San Francisco, CA. 